\title{
Sequential methotrexate and 5-fluorouracil therapy for gastric cancer patients with peritoneal dissemination: a retrospective study
}

\author{
Makoto Tahara, Atsushi Ohtsu, Narikazu Boku, Fumio Nagashima, Manabu Muto, Yasushi Sano, \\ Motoki Yoshida, Kiyomi Mera, Shu-ichi Hironaka, Hisao Tajiri, and Shigeaki Yoshida \\ Division of Digestive Endoscopy and Gastrointestinal Oncology, National Cancer Center Hospital East, 6-5-1 Kashiwanoha, Kashiwa, \\ Chiba 277-8577, Japan
}

\begin{abstract}
Background. Most gastric cancer patients with peritoneal dissemination have been excluded from clinical studies because they usually have no measurable lesions. They also have a high risk of toxicity because of complications such as intestinal obstruction and ascites. We conducted a retrospective analysis to evaluate the efficacy and feasibility of sequential methotrexate (MTX) and 5-flurorouracil (5FU) therapy for this population.

Methods. This analysis was based on 56 consecutive chemotherapy-naive patients with confirmed peritoneal dissemination of gastric cancer who were being treated with sequential MTX/5FU. The therapy comprised a weekly schedule of MTX $100 \mathrm{mg} / \mathrm{m}^{2}$, given as a bolus infusion $3 \mathrm{~h}$ prior to a bolus infusion of $5 F U 600 \mathrm{mg} / \mathrm{m}^{2}$. Leucovorin $10 \mathrm{mg} / \mathrm{m}^{2}$ was administered six times, every $6 \mathrm{~h}$, starting $24 \mathrm{~h}$ after MTX administration.

Results. Evidence of peritoneal dissemination was confirmed by laparotomy in 16 patients, by cytologic examination of ascites in 11 patients, and by clinical imaging in 29 patients (15 with ascites, 13 with intestinal obstruction; in 10 of the 29 patients, detection was by barium enema or computed tomography [CT] scan). Neutropenia of grade 3 or worse and anemia were observed in $8(14 \%)$ and $10(18 \%)$ of the 56 patients, respectively. There was one treatment-related death due to neutropenic sepsis. Of the 26 patients with measurable lesions, 9 showed a response $(36 \%)$. The median survival time and median time to treatment failure were 259 days and 167 days, respectively. Objective improvement of ascites was seen in 13 of 26 patients $(50 \%)$, including 5 with showed complete disappearance of ascites. Seven of the 15 patients $(47 \%)$ with intestinal obstruction showed resolution, and 8 of the 21 patients $\mathbf{( 3 8} \%)$ who needed nutritional support before the treatment were free of that support for a median duration of 220 days after the completion of the treatment. Forty-seven of the 56 patients $(84 \%)$ could be treated at outpatient clinics.
\end{abstract}

Offprint requests to: M. Tahara

Received: August 24, 2001 / Accepted: October 19, 2001
Conclusions. This regimen may be of clinical benefit for patients with peritoneal dissemination of gastric cancer.

Key word Gastric cancer · Peritoneal dissemination · Sequential MTX/5FU therapy

\section{Introduction}

Peritoneal dissemination is a major event in the development of gastric cancer [1], and causes many serious complications, such as intestinal obstruction, malignant ascites, and hydronephrosis. The symptoms of these events include abdominal pain, sensations of abdominal fullness, vomiting, and constipation, leading to an extremely poor quality of the patient's remaining life. Conservative management, with a nasogastric tube, antiemetics, or high-dosage steroids, is relatively ineffective. Although intraperitoneal chemotherapy has been used to treat peritoneal dissemination, the results remain poor [2-6].

Recent phase II studies of systemic chemotherapy have demonstrated high antitumor effects in the treatment of advanced gastric cancer [7-10]. However, most patients with peritoneal dissemination have been excluded from clinical studies because they rarely have measurable lesions and because they have a high risk of toxicity due to complications such as intestinal obstruction and ascites. Therefore, the efficacy of systemic chemotherapy for patients with peritoneal dissemination remains unclear.

Of several biochemical agents examined, the sequential use of the drugs methotrexate (MTX) and 5fluorouracil (5FU; sequential MTX/5FU) was first shown to be clinically effective against malignancies in the gastrointestinal tract [11]. This combination has also been reported to be effective in the treatment of various types of carcinomas, including gastric cancer. Konishi et 
al. [12] reported that this regimen showed higher efficacy in patients with a diffuse type of adenocarcinoma, defined histologically, than in those with an intestinal type. The response rate of patients with peritoneal dissemination in their study was $23 \%(6 / 26)$, and ascites was eliminated in 8 of 16 patients $(50 \%)$. From their findings, this regimen would be expected to improve the condition of advanced gastric cancer patients with peritoneal dissemination. However, the efficacy of this therapy has not been fully clarified.

In this retrospective study, we therefore investigated the feasibility and efficacy of sequential MTX/5FU therapy in gastric cancer patients with peritoneal dissemination. In addition, we also analyzed the responses of ascites and intestinal obstruction to the therapy, and the incidence of freedom from the need for nutritional support after the chemotherapy, to assess the patients' quality of life (QOL).

\section{Patients and methods}

\section{Patient selection}

Between August 1993 and December 1999, 95 patients with confirmed peritoneal dissemination of unresectable or recurrent gastric cancers were treated with sequential MTX/5FU therapy at the National Cancer Center Hospital East, Kashiwa, Japan. We selected patients from the database who fulfilled the following criteria: (i) no prior chemotherapy; (ii) definite evidence of peritoneal dissemination; (iii) 75 years old or less, with a Eastern Cooperative Oncology Group (ECOG) performance status (PS) of 2 or better; (iv) adequate bone marrow, renal, and hepatic functions; and (v) no serious medical complications. Patients with intestinal obstruction or ascites were included in this analysis. Of the 95 patients, 23 with prior chemotherapy, 3 with performance status 3, 2 with hyperbilirubinemia, 8 with disseminated intravascular coagulation (DIC), and 3 patients with renal dysfunction were excluded from this study.

\section{Treatment schedule}

The treatment schedule comprised the weekly administration of MTX $\left(100 \mathrm{mg} / \mathrm{m}^{2}\right.$, as an i.v. bolus $)$ followed $3 \mathrm{~h}$ later by $5 \mathrm{FU}\left(600 \mathrm{mg} / \mathrm{m}^{2}\right.$, i.v. bolus). From $24 \mathrm{~h}$ after the administration of MTX, calcium leucovorin $\left(10 \mathrm{mg} / \mathrm{m}^{2}\right.$, p.o. or i.v.) was administered every $6 \mathrm{~h}$, six times. To reduce the adverse effects of MTX, acetazolamide $(250 \mathrm{mg})$ was given intravenously immediately after the infusion of MTX, and sodium bicarbonate (33.2 mEq) added to $500 \mathrm{ml}$ of electrolyte solution was administered for urine alkalinization by drip infusion during the 3-h interval between the administration of MTX and 5FU. In principle, treatments were repeated weekly for three or four courses, followed by 1 -week rest. This treatment was continued until the disease progressed, or until the development of unacceptable toxicity, or until the patient refused further treatment. If there were adverse reactions on the day of administration, the treatment was postponed until the patient recovered.

\section{Diagnosis of peritoneal dissemination}

"Peritoneal dissemination" was defined when patients had at least one of the following findings: (i) peritoneal metastasis diagnosed by barium enema, computed tomography (CT) scan, or laparotomy; (ii) malignant ascites confirmed by cytologic examination, CT scan, or ultrasonography (US); or (iii) complete or incomplete intestinal obstruction confirmed by abdominal X-rays.

\section{Evaluation of antitumor effects and adverse events}

Objective responses in measurable metastatic lesions were evaluated according to standard WHO criteria [13]. The response for primary sites was assessed according to the response criteria of the Japanese Research Society for Gastric Cancer, based on roentgenographic and endoscopic findings [14]. The response of primary sites was not considered for the evaluation of the overall response. Improvement of ascites was defined if an objective decrease or disappearance was observed by CT scans and/or US. Improvement of intestinal obstruction was declared when the obstructive findings were resolved on abdominal $\mathrm{X}$-rays and the symptoms, such as abdominal pain, sensations of abdominal fullness, or vomiting, were relieved. Adverse events were evaluated according to the common toxicity criteria of the National Cancer Institute [15].

\section{Statistical analysis}

"Time to treatment failure" was calculated from the date of the first administration of sequential MTX/5FU therapy to the date at which the treatment was discontinued for any reason, or to the date of death from any cause during the treatment. The overall survival time was determined from the date of the first administration of sequential MTX/5FU therapy to the date of death by any cause, or to the last date of confirmed survival. Survival analysis was performed using the method of Kaplan and Meier [16].

\section{Results}

\section{Patient characteristics}

Fifty-six patients were selected as the subjects of this study. The patient's characteristics are listed in Table 1. The 56 patients had a median age of 58 years (range, 26- 
74 years) and a PS score of 0 in 29 patients, 1 in 17 patients, and 2 in 10 patients. Forty-five patients (80\%) had diffuse-type carcinomas, and 10 (18\%) had an intestinal-type histology. Gastrectomy had been performed in 30 patients. Twenty-five patients $(45 \%)$ had

Table 1. Patient characteristics

\begin{tabular}{lc}
\hline & No. of patients (\%) \\
\hline Total no. of patients & 56 \\
Age (years) & \\
Median & 58 \\
Range & $26-74$ \\
Sex & \\
Male & $35(63)$ \\
Female & $21(37)$ \\
Performance status & \\
0 & $29(52)$ \\
1 & $17(30)$ \\
2 & $10(18)$ \\
Histology & \\
Intestinal & $10(18)$ \\
Diffuse & $45(80)$ \\
Unknown & $1(2)$ \\
Surgical resection & \\
No & $26(46)$ \\
Yes & $30(54)$ \\
Metastatic site & $1(2)$ \\
Lung & $10(18)$ \\
Liver & $26(46)$ \\
Lymph node & $2(4)$ \\
Bone & $26(46)$ \\
Complication & $15(27)$ \\
Ascites & $8(14)$ \\
Intestinal obstruction & \\
Hydronephrosis & \\
\hline
\end{tabular}

at least one measurable lesion. Ascites, intestinal obstruction, and hydronephrosis without renal dysfunction were detected in $26(46 \%), 15(27 \%)$, and $8(14 \%)$ patients, respectively. In the 56 patients, peritoneal dissemination was detected by laparotomy in $16(29 \%)$, by cytologic examination of ascites in $11(20 \%)$, and by clinical imaging in $29(52 \%)$. Eight patients had both ascites and intestinal obstruction.

\section{Adverse events}

A total of 848 courses of the treatment were administered, with a median of 14 courses per patient (range, 1 to 56 courses). The adverse events are summarized in Table 2. The major adverse events were neutropenia, and anemia. Grade 3 or worse neutropenia, anemia, thrombocytopenia, and hyperbilirubinemia were observed in $8(14 \%), 10(18 \%), 1(2 \%)$, and $3(5 \%)$ patients, respectively. One patient experienced grade 3 cardiac ischemia after five courses of the treatment, but this resolved after a few minutes without medication. Another patient who had both ascites and intestinal obstruction developed fatal neutropenic sepsis after the first course of the treatment. During the first month after the initial treatment, the dose was reduced in only one patient, in whom peritoneal dissemination was detected by barium enema, because of grade 3 nausea. Another patient was treated on a 2 -week-based schedule because of grade 2 nausea with the initial treatment.

\section{Responses and survival}

The responses to this therapy are summarized in Table 3 . In the 25 patients with measurable lesions, the overall

Table 2. Adverse events in sequential MTX/5FU therapy

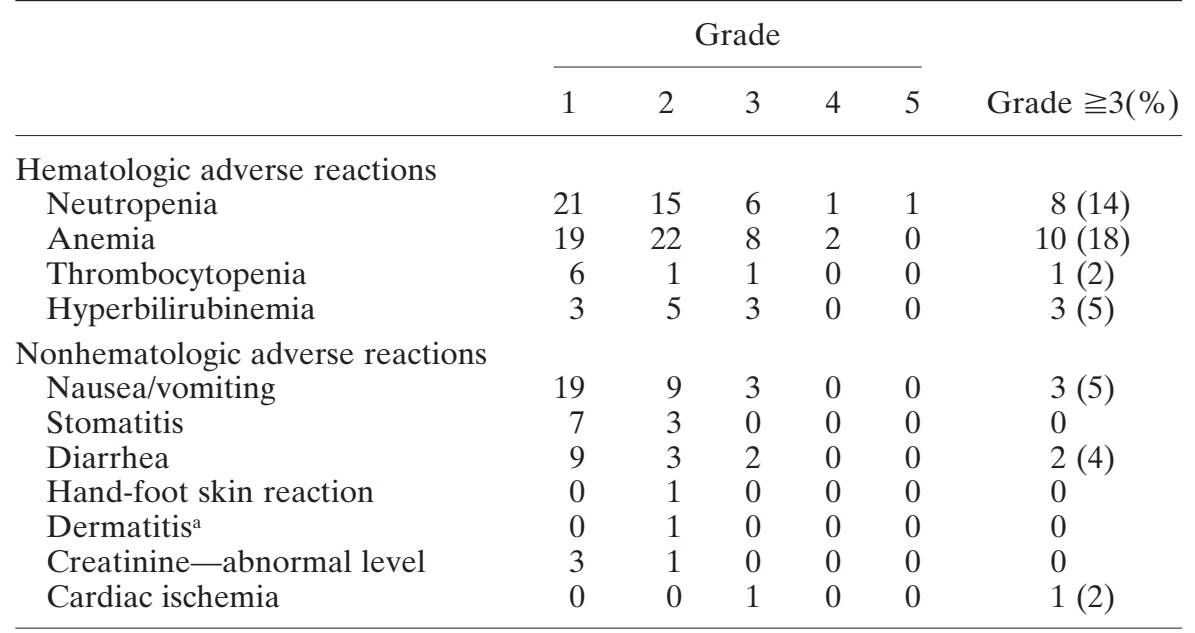

Grade was determined according to the common toxicity criteria of the National Cancer Institute [15]

MTX, Methotrexate; 5FU, 5-fluorouracil

${ }^{a}$ Herpes simplex virus infection 
Table 3. Response in patients with measurable lesions

\begin{tabular}{|c|c|c|c|c|c|c|}
\hline & \multirow[b]{2}{*}{ No. } & \multirow{2}{*}{$\frac{\mathrm{PR}}{\text { No. }(\%)}$} & \multirow{2}{*}{$\frac{\mathrm{NC}}{\text { No. (\%) }}$} & \multirow{2}{*}{$\frac{\mathrm{PD}}{\text { No. }(\%)}$} & \multirow{2}{*}{$\frac{\mathrm{NE}}{\text { No. }(\%)}$} & \multirow[b]{2}{*}{ Response rate } \\
\hline & & & & & & \\
\hline Overall & 25 & $9(36)$ & $9(36)$ & $4(16)$ & $3(12)$ & $36 \%$ \\
\hline Primary foci & 17 & $4(24)$ & $9(38)$ & $0(0)$ & $4(24)$ & $24 \%$ \\
\hline Lung & 1 & & $1(100)$ & & & $0 \%$ \\
\hline Liver & 11 & $5(45)$ & $2(18)$ & $2(18)$ & $2(18)$ & $45 \%$ \\
\hline Lymph node & 25 & $12(48)$ & $7(28)$ & $3(12)$ & $3(12)$ & $48 \%$ \\
\hline
\end{tabular}

PR, Partial response; NC, no change; PD, progressive disease; NE, not evaluable; No., number of patients

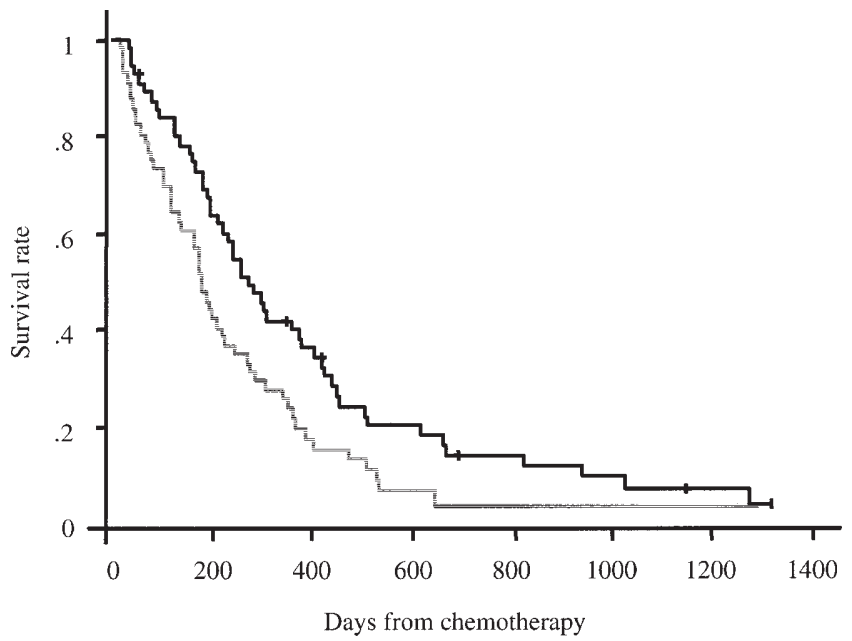

Fig. 1. Overall survival (continuous line) and time to treatment failure (dashed line) for 56 gastric cancer patients with peritoneal dissemination. With a median follow-up of 22 months, the median duration of survival and the median time to treatment failure of all patients were 259 days and 167 days, respectively

response rate was $36 \%(9 / 25)$. The response rates of patients with metastases in liver and lymph nodes were $45 \%$ (5/11), and 48\% (12/25), respectively. Objective improvement of ascites was seen in 13 of 26 patients $(50 \%)$. Of these, 5 showed the complete disappearance of ascites. Seven of the $15(47 \%)$ patients with intestinal obstruction showed the disappearance of the obstructive findings on abdominal X-ray, and the symptoms, such as abdominal pain, sensation of abdominal fullness, and vomiting, were relieved. With a median follow-up of 22 months, the median duration of survival and the median time to treatment failure of all patients were 259 days and 167 days, respectively (Fig. 1). The median survival times of the patients whose peritoneal dissemination was detected by laparotomy $(n=16)$ and those in whom this was detected by cytologic examination or by clinical imaging $(n=40)$ were 404 days and 244 days, respectively $(P=0.0447 ;$ Fig. 2$)$. The median survival times of the patients with $(n=21)$ and without

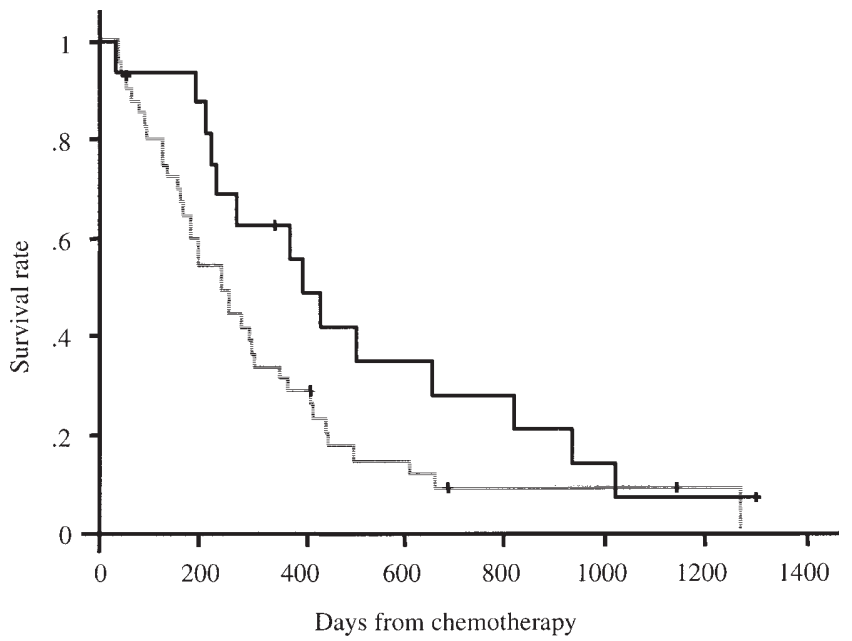

Fig. 2. Survival of the patients according to the method of diagnosis of peritoneal dissemination. Continuous line, Patients in whom peritoneal dissemination was detected by laparotomy $(n=16)$; dashed line, patients in whom peritoneal dissemination was detected by cytologic examination of ascites or by clinical imaging $(n=40)$. The median survival times of the patients in whom detection was achieved by laparotomy and by cytologic examination of ascites or clinical imaging were 404 days and 244 days, respectively $(P=$ $0.0447)$.

$(n=35)$ nutritional support were 196 days and 308 days, respectively $(P=0.0487$; Fig. 3$)$.

\section{Assessment of nutritional state}

Of the 21 patients who needed nutritional support before treatment, $8(38 \%)$ were free of this need after sequential MTX/5FU therapy. In these patients, the median duration without nutritional support was 220 days (range, 65-539 days). In the 35 patients without prior nutritional support, the median duration without the need for nutritional support was 178 days. Fortyseven of the 56 patients $(84 \%)$ could be treated at outpatient clinics. 


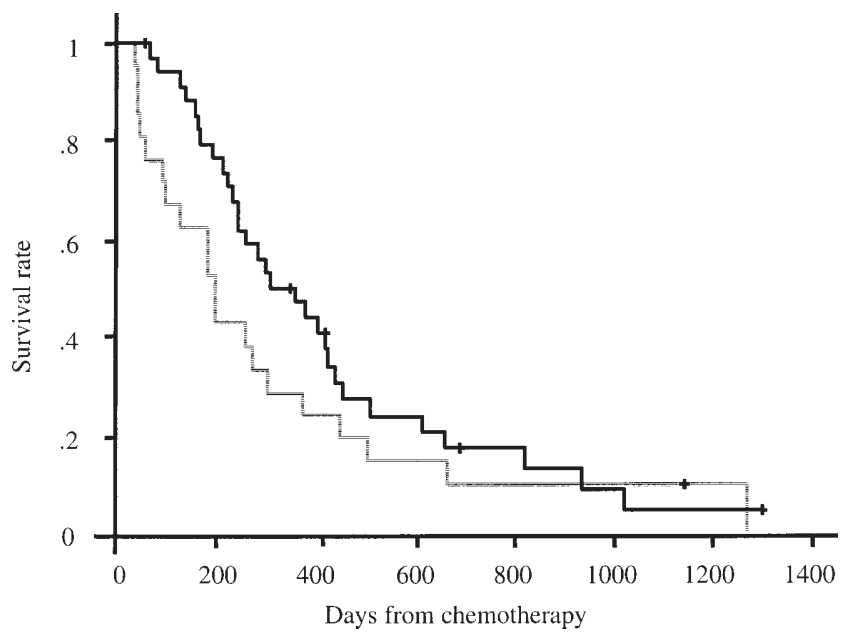

Fig. 3. Survival of the patients according to the need for nutritional support. Continuous line, Patients without need for nutritional support $(n=35)$; dashed line, patients with need for nutritional support $(n=21)$. The median survival times of the patients with and without nutritional support were 196 days and 308 days, respectively $(P=0.0487)$

\section{Discussion}

MTX penetrates slowly into third-space fluid collections, such as pleural effusion or ascites, reaching steady-state plasma concentrations in approximately $6 \mathrm{~h}$ $[17,18]$. The clearance of MTX from peritoneal fluid is about $5 \mathrm{ml} / \mathrm{min}$, substantially slower than its clearance from the plasma compartment, which equals or exceeds the glomerular filtration rate $(120 \mathrm{ml} / \mathrm{min})$. Third-space retention of an intravenously administered drug is associated with the prolongation of the terminal drug halflife in plasma, presumably owing to the slow reentry of the sequestered drug into the bloodstream [18]. This effect must be considered when treating patients with ascites or pleural effusions. Sequential MTX/5FU therapy generally has milder adverse effects than other combination chemotherapies. However, it was uncertain whether this regimen would be tolerable in those patients with retention of ascites or pleural effusion. Although there are no strict guidelines for dose adjustment in patients with third-space fluid accumulations, it is strongly recommended that this fluid be evacuated before treatment and that close monitoring of plasma drug concentrations is carried out in such patients. Konishi et al. [19] recommended a lower initial dose of $100 \mathrm{mg} /$ body to reduce the related risks for patients with massive ascites, based on their measurements of MTX serum levels. The present study demonstrated that, in none of the patients did plasma MTX concentrations exceed the level of $5 \times 10^{-7} \mathrm{M}$, which would require continued leucovorin rescue, during the routine monitoring of MTX concentrations in plasma $24 \mathrm{~h}$ after drug infusion without the evacuation of ascites or pleural effusion. Grade 3 neutropenia and diarrhea were observed in $5(20 \%)$ and $1(4 \%)$ of the 25 patients with ascites, respectively. Although one treatment-related death occurred, in a patient who had both ascites and intestinal obstruction, toxicity was generally mild in the other patients. Dose reduction was not performed in any of the 25 patients with ascites, while the dose of MTX/5FU was reduced in 1 patient, who had peritoneal dissemination detected by barium enema, because of grade 3 nausea. These results suggest that this intermediate-dose MTX $\left(100 \mathrm{mg} / \mathrm{m}^{2}\right)$ regimen may be tolerable even for those patients with ascites.

Few previous studies have revealed the safety of sequential MTX/5FU therapy for patients with intestinal obstruction. In the present study, grade 3 neutropenia and diarrhea were observed in $4(27 \%)$ and 1 (7\%) of the 15 patients with intestinal obstruction, respectively. Eight of the 15 patients with intestinal obstruction had ascites. In these 8 patients, grade 3 neutropenia and hyperbilirubinemia were observed in $4(50 \%)$ and 2 $(25 \%)$, respectively. Furthermore, the one treatmentrelated death occurred in a patient who had both ascites and intestinal obstruction. From these results, it appears that this regimen may be tolerable for patients with intestinal obstruction, but it should be administered more carefully in patients who have both ascites and intestinal obstruction than in patients with intestinal obstruction alone.

Intraperitoneal chemotherapy has been used to treat peritoneal dissemination, but the efficacy of this therapy remains poor [2-6]. This poor efficacy is believed to exist because the penetration distance of intraperitoneally injected drugs into the submesothelial tissue is limited to $2-3 \mathrm{~mm}[3,6]$, and because intraperitoneally injected drugs are usually distributed unevenly because of the anatomical complexity of the peritoneal cavity.

Systemic chemotherapy has been reported to have little effect on peritoneal dissemination, because of the existence of the peritoneum-plasma barrier, which limits drug penetration into the peritoneum [20,21]. Recently, sequential MTX/5FU therapy has been reported to be effective in patients with advanced gastric cancers [12,22-24]. The median survival time of advanced gastric cancer patients after this therapy has been reported to be approximately 7 months. Konishi et al. [12] reported that this regimen showed higher efficacy in patients with undifferentiated adenocarcinomas then in those with differentiated adenocarcinomas. The response rate of patients with peritoneal dissemination was $23.1 \%(6 / 26)$, and ascites was eliminated in 8 of 16 patients $(50 \%)$. In the present study, the response rate in patients with ascites was $36 \%(9 / 25)$, and the median survival time of all patients was 259 days. This result seems to be superior to that of the study of Konishi et al. 
[12]. However, the present study was a retrospective one and included patients with peritoneal dissemination detected only by laparotomy. Most of these patients had no measurable lesions and no severe complications such as ascites and intestinal obstruction. In addition, these patients had significantly better survival than those patients in whom peritoneal dissemination was detected by cytologic examination or clinical imaging (median survival times, 404 days and 244 days, respectively).

With the limited survival benefit for patients with advanced gastric cancer, QOL has been considered as another relevant end-point of chemotherapeutic trials in these patients. Peritoneal dissemination causes many serious complications, such as intestinal obstruction and malignant ascites. The symptoms of these events are upsetting, and include abdominal pain, sensations of abdominal fullness, vomiting, and constipation, which usually lead to hospital admission. In the present study, we investigated the response rate to the therapy of patients with ascites and intestinal obstruction, the rate of freedom from the need for nutritional support, and the rate of treatment at outpatient clinics after chemotherapy. Objective improvement of ascites was seen in $13 / 26(50 \%)$ patients. Of these 13 patients, 5 showed the complete disappearance of ascites. Seven of the 15 (47\%) patients with intestinal obstruction showed the disappearance of the obstructive findings on abdominal X-ray. Eight of the 21 patients (38\%) with nutritional support became free of such support and in these patients, the median duration without nutritional support was 220 days. In the 35 patients without prior need for nutritional support, the median duration without nutritional support was 178 days. These results suggest that this regimen may reduce the impairment of oral intake. In addition, most patients (84\%) could continue the treatment at outpatient clinics. In terms of QOL, this chemotherapy, which permits long-term use on an outpatient basis and alleviates complications such as ascites and intestinal obstruction, may be suitable and effective for advanced gastric cancer patients with peritoneal dissemination.

From the results of the present study, it appears that this regimen may not only have antitumor activity but that it may also have palliative potential, with tolerable toxicity. In conclusion, the results of this study are promising and warrant further investigation of this regimen of MTX/5FU to validate its clinical utility.

\section{References}

1. Dupont JB Jr, Lee JR, Burton GR, Cohn I Jr. Adenocarcinoma of the stomach: review of 1497 cases. Cancer 1987;41:941-7.

2. Hagiwara A, Takahashi T, Kojima O, Sawai K, Yamaguchi T, Yamane T, et al. Prophylaxis with carbon-absorbed mitomycin against peritoneal recurrence of gastric cancer. Lancet 1992;339: 629-31.

3. Koga S, Izumi A, Maeta M, Shimizu N, Osaki Y, Kanayama H. The effects of total-body hyperthemia combined with advanced cancer patients. Cancer 1983;52:1173-7.

4. Markman M. Intraperitoneal chemotherapy. Semin Oncol 1991; 18:248-54.

5. Brenner DE. Intraperitoneal chemotherapy: a review. J Clin Oncol 1986;4:1135-47.

6. Nakajima T. Tabular analysis of 10000 cases of gastric cancer. Jpn J Cancer Chemother 1994;21:1813-97.

7. Ajani JA, Foldor M, Van Cutsem E, Tjulandin S, Moiseyenko V, Cabral F, et al. Multinational randomized phase II trial of docetaxel (T) and cisplatin (C) with or without 5-fluorouracil $(5 \mathrm{FU})$ in patients with advanced gastric or GE junction adenocarcinoma (AGC-AGEJC) (abstract 957). Proc Am Soc Clin Oncol 2000;19:247.

8. Roth AD, Maibach R, Martinelli G, Fazio N, Aapro MS, Pagani $\mathrm{O}$, et al. Docetaxel (Taxotere)-cisplatin (TC): an effective drug combination in gastric carcinoma. Swiss Group for Clinical Cancer Research (SAKK), and the European Institute of Oncology. Ann Oncol 2000;11:301-6.

9. Boku N, Ohtsu A, Shimada Y, Shirao K, Seki S, Saito H, et al. A phase II study of a combination of irinotecan and cisplatin against metastatic gastric cancer. J Clin Oncol 1999;17:1-5.

10. Sakata Y, Ohtsu A, Horikoshi N, Sugimachi K, Mitachi Y, Taguchi T. Late phase II study of novel oral fluoropyrimidine anticancer drug S-1(1 M tegafur-0.4M Gimestat-1M Otastat potassium) in advanced gastric cancer. Eur J Cancer 1998;34: 1715-20.

11. Bertino JR, Wawicki WL, Lindquist CA, Guputa NS. Scheduledependent antitumor effects of methotrexate and 5-fluorouracil. Cancer Res 1977;37:327-8.

12. Konishi T, Hiraishi M, Mafune K, Miyama T, Hirata T, Mori K, et al. Therapeutic efficacy and toxicity of sequential methotrexate and 5-fluorouracil in gastric cancer. Anticancer Res 1994;14:127780 .

13. World Health Organization. WHO handbook for reporting results of cancer treatment. Geneva: WHO Offset Publication No.48, World Health Organization; 1979.

14. Japanese Research Society for Gastric Cancer. General rules for gastric cancer study. 11th ed. Tokyo: Kanehara, 1985.

15. National Cancer Institute. Common toxicity criteria. Bethesda: National Cancer Institute, Division of Cancer Treatment; 1998.

16. Kaplan EL, Meier P. Nonparametric estimation from incomplete observations. J Am Stat Assoc 1958;53:457-81.

17. Wan SH, Huffman DH, Azarnoff DL, Stephens R, Hoogstraten B. Effect of route of administration and effusion on methotrexate pharmacokinetics. Cancer Res 1974;34:3487-91.

18. Chabner BA, Stoller RG, Hande K, Jacobs S, Young RC. Methotrexate disposition in humans: case studies in ovarian cancer and following high-dose infusion. Drug Metab Rev 1978;8: 107-17.

19. Konishi T, Noie T, Yoshida J, Mafune K, Makuuchi M. Treatment of peritoneal dissemination of gastric cancer with sequential methotrexate and 5-fluorouracil. Gastric cancer 1999;2: $52-6$.

20. Los G, Mutsaers PH, van der Vijgh WJ, Baldew GS, de Graaf PW, McVie JG. Direct diffusion of cis-diamminedichloroplatinum(II) in intraperitoneal rat tumors after intraperitoneal chemotherapy: a comparison with systemic chemotherapy. Cancer Res 1989;49:3380-4.

21. Sugarbaker PH, Stuart A, Vidal-Jove J, Pessagno AM, Debruijin EA. Pharmacokinetics of the peritoneal plasma barrier after systemic mitomycin C administration. In: Sugarbaker PH, (ed). Peritoneal carcinomatosis: principles of management. Boston: Kluwer Academic; 1994. pp. 41-51.

22. Perez JE, Lacava JA, Dominguez ME, Rodriguez R, Barbieri MR, Ortiz EH, et al. Biochemical modulation of 5-fluorouracil by 
methotrexate in patients with advanced gastric carcinoma. Am J Clin Oncol 1998;21:452-7.

23. Dickinson R, Preagrave P, Levi J, Milliken S, Woods R. Sequential moderate-dose methotrexate and 5-fluorouracil in advanced gastric adenocarcinoma. Cancer Chemother Pharmacol 1989;24: $67-8$.
24. Sasaki T, Ota K, Ibayashi J, Sakata Y, Matsuoka T, Ishikawa M, et al. Randomized multicenter trial of sequential methotrexate and 5-fluorouracil versus 5-fluorouracil alone in advanced gastric cancer (in Japanese with English abstract). Jpn J Cancer Chemother 1989;16:2545-55. 